\title{
Estrategias de afrontamiento del ciberacoso desde la perspectiva de la víctima en adolescentes mexicanos de secundaria y bachillerato
}

\author{
Coping with cyberbullying strategies from the victim's perspective \\ in Mexican adolescent Junior and High School students \\ Josefina Sandoval Martínez \\ Alejandro César Antonio Luna Bernal \\ Alejandra De Gante Casas
}

\begin{abstract}
RESUMEN
El Inventario de Estrategias de Afrontamiento del Ciberacoso desde la perspectiva de la Víctima (IEAC-V) es un instrumento que mide el grado en que los adolescentes se inclinan a utilizar siete tipos de estrategias para hacer frente a posibles situaciones de ciberacoso en caso de llegar a ser víctimas: estrategias agresivas, denunciar, ignorar y desviar la preocupación, buscar apoyo, reunir pruebas, evitar al agresor y desconectarse. El presente trabajo aporta nueva información relevante acerca de la validez de este instrumento a través de un análisis factorial confirmatorio. La muestra se conformó por 992 adolescentes estudiantes de secundaria y bachillerato con edades de 12 a 20 años. Los resultados mostraron adecuadas cargas factoriales, coeficientes de determinación e índices de bondad de ajuste. Se encontraron también adecuados índices de confiabilidad así como diferencias estadísticamente significativas por género, edad y grado escolar. Se discuten estos y otros resultados en el contexto de la literatura psicológica sobre estrategias de afrontamiento del ciberacoso en la adolescencia.
\end{abstract}

Palabras clave: acoso cibernético, adolescentes, análisis factorial confirmatorio, test psicométrico, violencia escolar.

\begin{abstract}
The Coping with Cyberbullying Strategies from the Victim's perspective Inventory (Inventario de Estrategias de Afrontamiento del Ciberacoso desde la perspectiva de la Víctima, IEAC-V) is an instrument that measures the degree to which adolescents tend to use seven types of strategies to deal with possible cyberbullying situations, in the event of becoming victims: aggressive strategies, reporting, ignoring and deflecting concern, seeking support, gather evidence, avoid the bully, and to stop using social networks or the internet. The present paper provides new relevant information about the validity of this instrument through a confirmatory factor analysis. The sample was composed of 992 adolescent Junior and High School students, aged 12 to 20 years. The results showed factor loadings, coefficients of determination and adjustment values adequate. There were also adequate reliability indices as well as statistically significant differences by gender, and school grade. These and other results are discussed in the context of the psychological literature on coping with cyberbullying strategies in adolescence.
\end{abstract}

Keywords: cyberbullying, adolescents, confirmatory factor analysis, psychological tests, school violence. 


\section{INTRODUCCIÓN}

Actualmente las nuevas generaciones se han desarrollado en estrecho contacto con las tecnologías de información y comunicación (TIC), están conectadas la mayor parte del día, siendo esta una forma muy común de comunicación entre los adolescentes la cual realizan a través de diversos medios tecnológicos. Si bien esta situación tiene indudables aspectos positivos, es sabido que también ha llevado al surgimiento de diversos problemas, tales como las conductas de acoso e intimidación en el ciberespacio (Baldry, Farrington y Sorrentino, 2015).

El ciberacoso es un acto de agresión que se comete por medio de las TIC deliberadamente y puede ser perpetrado por un grupo o un sujeto mediante el uso de formas electrónicas de contacto, de manera recurrente durante un lapso de tiempo, en contra de una persona que es considerada víctima, y que no se puede defender por sí misma (Beltrán-Catalán, Zych, Ortega-Ruiz y Llorent, 2018; Gradinger, Yanagida, Strohmeier y Spiel, 2015; Smith, Mahdavi, Carvalho, Fisher, Russell y Tippett, 2008).

$\mathrm{Al}$ ciberacoso se le tipifica como un acto nocivo de violencia (on line) al igual que el bullying tradicional (off line), y ambos comparten ciertos rasgos como por ejemplo la intencionalidad, la repetición y la asimetría de poder. Una característica adicional es que puede ser realizado a cualquier hora desde cualquier lugar desde dispositivos móviles de mano y da la posibilidad de hacer ataques las 24 horas del día, los 7 días de la semana. La virtualidad aumenta el potencial de una mayor audiencia y el agresor puede hostigar, denigrar y pasar como anónimo, invadir lugares privados considerados seguros o hasta engañar al simular otra identidad para causar daño a sus pares (Herrera-López, Romera y Ortega-Ruiz, 2018; Li, 2008).

El ciberacoso se presenta en diversas regiones del planeta (Herrera-López, Romera y Ortega-Ruiz, 2017; Lee y Shin, 2017; Ortega, Del Rey y Casas, 2016; Waasdorp y Bradshaw, 2015) y puede llegar a convertirse en un grave problema global (Li, 2008).

Josefina Sandoval Martínez. Profesora titular de tiempo completo adscrita al Departamento de Psicología Aplicada de la Universidad de Guadalajara, México. Es doctora en Psicología por la Universidad de Guadalajara, integrante del Cuerpo Académico Violencia, Manejo de Conflicto y Mediación (UDG-CA-705) y coordinadora de Planeación del Departamento de Psicología Aplicada. Cuenta con reconocimiento al perfil deseable Prodep. Correo electrónico: josefina.sandoval@academicos.udg.mx. ID: https://orcid.org/0000-0002-4113-1897.

Alejandro César Antonio Luna Bernal. Profesor-investigador del Departamento de Filosofía de la Universidad de Guadalajara, México. Es doctor en Psicología por la Universidad de Colima. Tiene los reconocimientos al perfil deseable ProdeP y del Sistema Nacional de Investigadores, Nivel 1. Miembro del Cuerpo Académico Adolescentes: Mundo y Vida (UDG-CA-967) de la Universidad de Guadalajara. Correo electrónico: alejandro.luna@academicos.udg.mx. ID: https://orcid.org/0000-0002-3371-5999.

Alejandra De Gante Casas. Doctoranda en Psicología por la Universidad de Guadalajara, México. Profesora-investigadora del Departamento de Psicología Aplicada de la Universidad de Guadalajara. Miembro del Cuerpo Académico de Violencia, Manejo de Conflicto y Mediación (UDG-CA-705). Cuenta con reconocimiento al perfil deseable Prodep. Correo electrónico: alejandra. degante@academicos.udg.mx. ID: https://orcid.org/0000-0001-7186-1359. 
Múltiples estudios a nivel mundial revelan datos de gran magnitud, ya que uno de cada tres menores se encuentra involucrado en alguna situación de bullying y uno de cada cinco en ciberacoso (Herrera-López, Romera y Ortega-Ruiz, 2018).

Algunos estudios señalan que en México cerca del 20\% de adolescentes participan como actores de acoso mediante ciberacoso (Lucio, 2014; Martínez-Vilchis, Pozas, Jiménez, Morales, Miranda, Delgado y Cuenca, 2015; Valdés, Carlos, Tánori y Wendlandt, 2014). Varias investigaciones refuerzan que es la adolescencia el periodo cuando mayormente se repite esta conducta (Beltrán-Catalán et al., 2018; Gradinger et al., 2015). Por otra parte, en una revisión sistemática llevada a cabo por Vega-Cauich (2019) se examinaron 27 estudios sobre acoso y ciberacoso escolar en muestras mexicanas, llegando a la conclusión de que hay una prevalencia de bullying de 20\% para víctimas y $19 \%$ para agresores, así como una prevalencia de ciberacoso de $21 \%$ para cibervíctimas y $11 \%$ para ciberagresión en nuestro país.

Actualmente existe a nivel mundial una gran preocupación social ya que por su alta prevalencia el ciberacoso puede llegar a convertirse en un serio problema global (Li, 2008) debido a las graves consecuencias de las experiencias de ciberacoso sobre la salud psicológica y social de niños, niñas, adolescentes y jóvenes (León, Felipe, Fajardo y Gómez-Carroza, 2012; Matos, Vieira, Amado, Pessoa y Martins, 2018). Estudios reportan que los adolescentes víctimas de ciberacoso sufren laceraciones psicológicas, emocionales y sociales tales como miedo, irritabilidad, baja autoestima, falta de concentración, o el desarrollo de síntomas de depresión, somatizaciones y ansiedad fóbica que pueden repercutir en la vida adulta (Del Rey, Estévez y Ojeda, 2018; Ferrara, Ianniello, Villani y Corsello, 2018; Garaigordobil, 2011; Redondo, Luzardo-Briceño, García-Lizarazo e Inglés, 2017; Schneider, O’Donnell, Stueve y Coulter, 2012).

En ese contexto, recientemente se ha propuesto orientar las investigaciones hacia el estudio de las estrategias de afrontamiento de los individuos frente al ciberacoso, debido a que diversos autores han mostrado que los efectos de las experiencias de ciberacoso podrían ser moderados por el modo en que los sujetos hacen frente a dichas situaciones (p.e., Jacobs, Vollink, Dehue y Lechner, 2015; Machmutow, Perren, Sticca y Alsaker, 2012; Ortega-Barón y Carrascosa, 2018; Raskauskas y Huynh, 2015). En este orden de ideas, las diferencias encontradas entre los individuos en los efectos del ciberacoso sobre el bienestar podrían deberse al uso y la efectividad de las estrategias de afrontamiento empleadas por ellos (Sticca, Machmutow, Stauber, Perren, Palladino, Nocentini, Menesini, Corcoran y McGuckin, 2015), ya que estas atraen consecuencias positivas o negativas, e influyen en la posibilidad de experimentar la situación como estresante o inhibir la sensación de estrés (Torres-Acuña, Rivera y Navarro, 2019). Debido a lo anterior, y dada la escasez de estudios a este respecto, la presente investigación pretende ser una contribución a esta área de investigación 
sobre las estrategias de afrontamiento ante el ciberacoso empleadas por los adolescentes mexicanos estudiantes de secundaria y bachillerato.

Lazarus y Folkman (1984) definen el afrontamiento (coping) como "los esfuerzos cognitivos y conductuales constantemente cambiantes para manejar las demandas externas y/o internas específicas que son evaluadas como gravosas o excedentes a los recursos de la persona” (p. 141). Aplicando este planteamiento al ciberacoso (entendido como una situación estresante para la víctima), el estudiante comienza el afrontamiento en primer término con la evaluación de las demandas que plantea la situación de asedio, en segundo término valora los recursos con que cuenta para enfrentarla, y entonces selecciona una estrategia frente a ella (Jacobs et al., 2015; Mallmann, Macedo y Zanatta, 2018).

El modelo transaccional del afrontamiento ante el estrés de Lazarus y Folkman (1984) plantea que existen dos tipos de estrategias generales de afrontamiento: 1) orientada al problema, la cual se dirige a mejorar la situación generadora de estrés, y 2) enfocada en la emoción, la cual se centra en modificar el estado emocional del sujeto. Alipan, Skues y Theiler (2018) mostraron que los individuos que utilizan el afrontamiento orientado al problema en situaciones de acoso cibernético tienden a tener resultados más positivos y adaptativos con respecto a quienes usan estrategias situadas en la emoción o de evitación.

Algunas estrategias ante el ciberacoso han sido estudiadas a nivel internacional con base en esta teoría general del afrontamiento (p. e., Mallmann, Macedo y Zanatta, 2018; Nacimiento y Mora-Merchán, 2014; Palladino, Nocentini y Menesini, 2012). Estos enfoques generales están fundamentados en la suposición de que la forma en que las personas enfrentan el estrés en general es coherente con la manera en que se manejan las situaciones de ciberacoso (Alipan, Skues y Theiler, 2018). Aunque se reconoce el aporte de estos acercamientos, estudiosos del ciberacoso insisten en la necesidad de realizar abordajes más específicos dada su complejidad y llaman la atención para contar con modelos e instrumentos que midan un mayor número de dimensiones (Jacobs et al., 2015; Machackova, Cerna, Sevcikova, Dedkova y Daneback, 2013; Sticca et al., 2015).

En este sentido, con el fin de identificar los diferentes tipos de estrategias de afrontamiento empleadas frente al ciberacoso algunas investigaciones han utilizado preguntas abiertas ya sea con adolescentes (p.e., Garaigordobil, 2013; Lucio y Gómez, 2018) o con estudiantes universitarios (p.e., Bezerra, Veiga y Caetano, 2014), procediendo a categorizar las respuestas de los participantes; por ejemplo, el estudio de Giménez Gualdo (2015) clasifica las respuestas en cuatro tipos: asertivas, búsqueda de ayuda, reciprocidad negativa, y pasivas, no obstante aclara que es solo para fines expositivos. Algunos estudios realizados mediante entrevistas (Parris, Varjas, Meyers y Cutts, 2012; Sléglová y Cerna, 2011; Weber y Pelfrey Jr., 2014) emplean estrategias 
metodológicas similares, elaboran categorías con base en los discursos de los participantes; por ejemplo, Parris et al. (2012) obtuvieron cuatro categorías de estrategias: evitación, aceptación, justificación y búsqueda de soporte social. Por otra parte, se encuentran también las investigaciones que se han propuesto diseñar escalas psicométricas para medir estrategias de afrontamiento del ciberacoso (De Gante-Casas, Luna-Bernal, López-Aguilar y Nava-Preciado, 2020; Jacobs et al., 2015; Machmutow et al., 2012; Ronis y Slaunwhite, 2019; Sticca et al., 2015). Este tipo de estudios centran su interés en proporcionar datos acerca de la validez y confiabilidad de los instrumentos, y obtienen la clasificación de las diversas estrategias de afrontamiento a través de un proceso de validación basado en el análisis factorial. En la presente investigación se consideró adecuado proseguir con esta vía metodológica, considerando que el análisis de la estructura factorial contribuye también a las reflexiones sobre la validez y naturaleza de los constructos (Ferrando y Anguiano-Carrasco, 2010; Moral, 2016).

La investigación sobre estrategias de afrontamiento del ciberacoso es escasa en México hasta el momento. La revisión bibliográfica realizada para el presente trabajo mostró dos estudios sobre estrategias de afrontamiento frente al bullying tradicional realizados mediante adaptaciones de instrumentos que evalúan afrontamiento en general. Uno de ellos fue llevado a cabo con una muestra $(N=130)$ de docentes de secundarias particulares de la Ciudad de México (Elizalde, 2010) y otro con 1,170 estudiantes adolescentes de nivel medio superior matriculados en 35 planteles en Nuevo León (Madrid, Valdéz y Vera, 2015). También se identificaron estudios que informan de manera incidental sobre estrategias empleadas por adolescentes ante la ciberagresión, ya que no era su objetivo central y además los estudios fueron a través de preguntas abiertas (Lucio y Gómez, 2018; Vega, González-Pérez y Quintero-Vega, 2013).

En cuanto a trabajos que diseñen instrumentos psicométricos y los validen a través del análisis factorial, se identificaron tres estudios realizados en México (De Gante-Casas et al., 2020; Luna-Bernal, Gómez-Pérez, Sandoval-Martínez y ValenciaAguirre, 2020; Torres-Acuña, Rivera y Navarro, 2019). El de Torres-Acuña, Rivera y Navarro (2019) fue llevado a cabo con una muestra de 346 estudiantes de la carrera de Educación de una universidad del sur de Sonora. Después de realizar el análisis factorial a su instrumento la versión final quedó conformada por 11 reactivos seccionados en tres factores: búsqueda de apoyo social, solución técnica evasiva y solución técnica proactiva.

Por su parte, el trabajo de De-Gante Casas et al. (2020) estuvo orientado a diseñar un Inventario de Estrategias de Afrontamiento del Ciberacoso desde la perspectiva del Observador (IEAC-O) en un estudio con una muestra de 526 adolescentes estudiantes de bachillerato. Dicho instrumento proporciona información acerca de las estrategias empleadas por los adolescentes no en su papel de víctimas de ciberacoso sino en el 
rol de observadores o testigos de ciberagresiones. En este estudio se identificaron seis estrategias mediante el análisis factorial exploratorio: denunciar los hechos ante una autoridad; ignorar lo que está pasando y desviar la preocupación; estrategias agresivas; reunir pruebas y buscar apoyo en una persona de confianza; evitación ansiosa o desconectarse de las redes sociales y el Internet, y evitar interactuar con los agresores.

Por su parte, Luna-Bernal et al. (2020) propusieron y analizaron las propiedades psicométricas de una versión de este mismo instrumento orientada a medir las estrategias de afrontamiento frente al ciberacoso empleadas por los adolescentes pero en su rol de víctimas de las ciberagresiones. El instrumento denominado Inventario de Estrategias de Afrontamiento del Ciberacoso desde la perspectiva de la Víctima (IEAC-V) en su versión original estuvo compuesto por 39 reactivos, cada uno de los cuales representa una estrategia concreta empleada por adolescentes. El estudio empírico se llevó a cabo con una muestra de 544 adolescentes estudiantes de bachillerato de dos planteles públicos ubicados en el estado de Jalisco (uno regional y uno metropolitano). Una vez realizado el análisis factorial exploratorio, la versión final del IEAC-V quedó compuesta por 31 reactivos divididos en siete escalas, las cuales se interpretan como diversos tipos de estrategias:

a. Estrategias agresivas o agresión: abarca aquellas estrategias empleadas por la víctima cuando intenta enfrentar al acosador de manera hostil, tales como Vengarme del agresory de quienes participaron; Amenazar a quienes me están acosando; Postear cosas negativas del agresor y de sus cómplices, e Insultar al agresor;

b. Denuncia: abarca acciones tendientes a informar los hechos ante una autoridad procurando su intervención, tales como Contárselo a un profesor; Acudir a la policía y denunciar a quien lo está haciendo; Contárselo al prefecto de disciplina; Contárselo al director de la escuela;

c. Ignorar y desviar la preocupación: incluye comportamientos dirigidos a controlar los propios pensamientos y emociones tratando de ignorar lo que está sucediendo, tales como Tomarlo con humor y pensar que no es tan grave; Intentar no pensar en lo que está pasando; Ignorar, no hacer caso de las agresiones, e Ignorar a los agresores;

d. Buscar apoyo: consistente en buscar ayuda y soporte en un familiar o una persona de confianza, tales como Contárselo a mi papá; Pedir ayuda a una persona de confianza y juntos pensar qué hacer al respecto; Contárselo a mi mamá, y Contárselo a algún familiar;

e. Pruebas: se refiere a conservar y guardar evidencias que den constancia de los hechos, como Hacer captura de pantalla (screenshot) para guardarlo como prueba y Guardar todo lo que pueda servir como prueba de lo que me están haciendo;

f. Evitar al agresor: orientada a utilizar medios tecnológicos y no tecnológicos para lograr no tener contacto con los agresores, sin necesidad de dejar de usar internet ni los sitios web de preferencia, tales como Borrar al agresor de los 
contactos; Evitar tener cualquier tipo de contacto con los agresores, y Bloquear al acosador en la cuenta (Facebook, Twitter, Instagram, etc.), y

g. Desconexión: que consiste en evadir la situación evitando completamente el uso de internet o de las páginas o sitios web relacionados con el problema, tales como Dejar de utilizar internet; Desactivar mi página de Facebook, Twitter, Instagram, etc., y Evitar todos los sitios web en que me agreden.

En el marco de lo anterior, el presente estudio pretende aportar nueva información relevante sobre la validez del IEAC-V, llevando a cabo un análisis factorial confirmatorio del instrumento. El análisis factorial confirmatorio permitirá poner a prueba el modelo hipotetizado de la estructura factorial del IEAC-V, obtenido a partir del análisis factorial exploratorio realizado en el estudio precedente de Luna-Bernal et al. (2020), así como evaluar la calidad del ajuste de dicho modelo a los datos mediante índices de bondad de ajuste. Debido a estas características es que el análisis factorial confirmatorio ha sido entendido en la literatura como una técnica que "corrige las deficiencias inherentes a la perspectiva exploratoria" (Batista-Foguet, Coenders y Alonso, 2004, p. 24), permitiendo así llegar a consideraciones más sólidas acerca de la validez de los instrumentos y de los constructos que los sustentan (Ferrando y Anguiano-Carrasco, 2010).

Además es importante considerar el hecho de que el mencionado estudio de Luna-Bernal et al. (2020) fue realizado con una muestra de estudiantes de bachillerato con edades de 15 a 19 años, mientras que la presente investigación se ha propuesto estudiar la estructura factorial del instrumento en una muestra de adolescentes de secundaria y bachillerato, ampliando así el rango de edad y de escolaridad.

Así, en el presente trabajo se planearon tres objetivos específicos: a) realizar un análisis factorial confirmatorio del IEAC-V, con el fin de evaluar su estructura factorial y su bondad de ajuste; b) estudiar la confiabilidad de cada una de las escalas del instrumento, y c) analizar posibles diferencias significativas por género, edad y grado escolar.

\section{MÉtodo}

\section{Participantes}

La muestra del presente estudio estuvo constituida por 992 adolescentes con rango de edad de 12 a 20 años $(M=15.92, D E=1.70)$. Los participantes eran estudiantes matriculados en seis planteles: dos de nivel secundaria $(n=198,20.0 \%)$ y cuatro de nivel bachillerato $(n=794,80.0 \%)$. Las escuelas participantes fueron elegidas de manera no probabilística y por conveniencia. Por su parte, la muestra de estudiantes se conformó eligiendo aleatoriamente un grupo de cada grado escolar en cada uno de los planteles escolares participantes. En la tabla 1 se presenta la distribución de los participantes por grupos de edad y grado escolar en función del género. Como 
se puede observar, los datos de nivel bachillerato (educación media superior) fueron agrupados por anualidades, de la siguiente manera: primer año (semestres 1 y 2), segundo año (semestres 3 y 4) y tercer año (semestres 5 y 6 ), ello con el fin de facilitar su análisis y comparación con los tres grados de secundaria.

Tabla 1. Distribución de la muestra por edad y grado escolar en función del género.

\begin{tabular}{lccc} 
& $\begin{array}{c}\text { Hombres } \\
n(\%)\end{array}$ & $\begin{array}{c}\text { Mujeres } \\
n(\%)\end{array}$ & $\begin{array}{c}\text { Total } \\
n(\%)\end{array}$ \\
\hline 12 a 15 años & $158(15.9)$ & $195(19.7)$ & $353(35.6)$ \\
\hline 16 y 17 años & $193(19.5)$ & $272(27.4)$ & $465(46.9)$ \\
\hline 18 a 20 años & $78(7.9)$ & $96(9.7)$ & $174(17.5)$ \\
\hline Primer año secundaria & $37(3.7)$ & $28(2.8)$ & $65(6.6)$ \\
\hline Segundo año secundaria & $30(3.0)$ & $37(3.7)$ & $67(6.8)$ \\
\hline Tercer año secundaria & $24(2.4)$ & $42(4.2)$ & $66(6.7)$ \\
\hline Primer año bachillerato & $120(12.1)$ & $166(16.7)$ & $286(28.8)$ \\
\hline Segundo año bachillerato & $102(10.3)$ & $144(14.5)$ & $246(24.8)$ \\
\hline Tercer año bachillerato & $116(11.7)$ & $146(14.7)$ & $262(26.4)$ \\
\hline Total & $429(43.2)$ & $563(56.8)$ & $992(100.0)$ \\
\hline
\end{tabular}

$N=992$. Los porcentajes de la tabla son con relación al total de la muestra.

Fuente: Construcción personal.

\section{Instrumento}

Inventario de Estrategias de Afrontamiento del Ciberacoso desde la perspectiva de la Víctima (IEAC-V).

Es un instrumento que mide el grado en que los adolescentes se inclinan a utilizar siete tipos de estrategias para hacer frente a posibles situaciones de ciberacoso en caso de llegar a ser víctimas: a) estrategias agresivas, b) denunciar, c) ignorar y desviar la preocupación, d) buscar apoyo en una persona de confianza, e) reunir pruebas, f) evitar al agresor y g) desconectarse. El formato de respuesta es una escala tipo Likert de cuatro puntos que va de $1=$ Definitivamente no haría eso, $2=$ Probablemente no haría eso, 3 = Probablemente sí haría eso, hasta 4 = Definitivamente sí haría eso. Para calificar el instrumento se calculó la media aritmética de los reactivos de cada una de las siete escalas.

La construcción y validación del IEAC-V fue llevada a cabo por Luna-Bernal et al. (2020) en un estudio realizado con 544 adolescentes estudiantes de bachillerato de dos planteles públicos ubicados en el estado de Jalisco, México. El instrumento original tenía 39 reactivos. Los autores llevaron a cabo un análisis factorial exploratorio obteniendo adecuadas cargas factoriales y porcentajes de varianza explicada. La versión final del instrumento quedó conformada por 31 reactivos. Los índices de 
confiabilidad alfa de Cronbach reportados por estos autores fueron los siguientes: estrategias agresivas (seis reactivos) $=.86$, denunciar $($ cinco reactivos $)=.83$, ignorar y desviar la preocupación (seis reactivos) $=.71$, buscar apoyo (cuatro reactivos) $=$ .81 , reunir pruebas (tres reactivos) $=.70$, evitar al agresor (cuatro reactivos) $=.72 \mathrm{y}$ desconectarse (tres reactivos) $=.70$.

En el presente trabajo se aplicó la versión original del instrumento compuesta por 39 reactivos, pero para el análisis de datos solo se utilizaron los 31 ítems correspondientes a la versión final de IEAC-V obtenida en el estudio mencionado de Luna-Bernal et al. (2020).

\section{Procedimiento}

Se obtuvo la colaboración de las instituciones educativas con las cuales se estableció contacto a través la unidad de Orientación Educativa y de la dirección de cada plantel, quienes otorgaron la autorización para el estudio una vez que se realizaron las consultas necesarias conforme a sus procedimientos internos. Obtenida la autorización se seleccionó al azar un grupo de cada grado en cada plantel educativo y se aplicó el instrumento dentro de cada aula, explicando a los estudiantes que su participación sería absolutamente voluntaria y anónima, que se haría un manejo confidencial de la información por parte de los investigadores y que dicha información sería utilizada exclusivamente para fines estadísticos y científicos.

\section{Análisis estadístico}

Con relación al primer objetivo del presente estudio, se realizó un análisis factorial confirmatorio (AFC) empleando el método de máxima verosimilitud (Maximum Likelihood, ML) junto con el procedimiento de bootstrapping, el cual está recomendado cuando se trata de escalas tipo Likert y los datos presentan un alejamiento de la curva normal (Byrne, 2010; Medrano y Muñoz-Navarro, 2017). Para ello se establecieron 500 replicaciones bootstrap e intervalos de confianza corregidos al 90\%. Para la especificación del modelo se tomó la composición factorial del IEAC-V reportada en el estudio de Luna-Bernal et al. (2020), considerando a los siete tipos de estrategias de afrontamiento como variables latentes relacionadas y a los correspondientes reactivos como las variables observadas.

Para evaluar la bondad de ajuste del modelo examinado se utilizaron los siguientes índices (Hair, Anderson, Tatham y Black, 1999):

Como indicadores de ajuste absoluto, el estadístico-ratio de verosimiltud Chicuadrado (Chi square), el índice de bondad de ajuste (Goodness of Fit Index, GFI ), el residuo cuadrático medio de aproximación (Root Mean Square Error of Approximation, RMSEA) y el residuo cuadrático medio en su modalidad estandarizada (Standardized Root Mean Square Residual, SRMR). 
Como indicadores de ajuste incremental el índice de ajuste normado (Normed Fit Index, NFI), el índice de bondad de ajuste comparativo (Comparative Fit Index, CFI), el coeficiente de Tucker-Lewis (TLI) y el índice de bondad de ajuste en su modalidad corregida (Adjusted Goodness of Fit Index, AGFI).

Finalmente, como indicadores de parsimonia se utilizaron el índice de bondad de ajuste de parsimonia (Parsimonious Goodness of Fit Index, PGFI), así como el cociente entre Chi-cuadrada y sus grados de libertad, también llamada Chi-cuadrado normado (Normed Chi-Square).

El estadístico de Chi-cuadrado es indicador de un buen ajuste si presenta niveles bajos y resulta no ser estadísticamente significativo, sin embargo debe tomarse en cuenta que se trata de un valor muy sensible al tamaño de la muestra, especialmente si su tamaño es mayor de 200 participantes (Hair et al., 1999). Debido a esta y otras limitaciones que ha presentado este índice, en la actualidad no se recomienda utilizarle "como prueba estadística, sino simplemente como una medida descriptiva de ajuste" (Medrano y Muñoz-Navarro, 2017, p. 228).

El RMSEA y el SRMR se consideran excelentes con valores menores a .05 (Byrne, 2010), pero indicarían un ajuste admisible entre .05 y .075 (Moral, 2016). Los índices GFI, AGFI, NFI, TLI y CFI cercanos a 1 indican excelente ajuste pero se consideran aceptables arriba de .90 (Byrne, 2010; Hair et al., 1999; Moral, 2016). Los puntajes de PGFI se consideran aceptables si son superiores a .50 siempre que vayan acompañados de adecuados valores absolutos e incrementales (Byrne, 2010), aunque Escobedo-Portillo, Hernández-Gómez, Estebané-Ortega y Martínez-Moreno (2016) ubican los valores entre .50 y .70. Finalmente, en cuanto al estadístico de Chi-cuadrado normado, los valores entre 2 y 5 indican un ajuste razonable (Hair et al., 1999).

Para realizar el segundo objetivo de esta investigación se obtuvo el coeficiente de confiabilidad alfa de Cronbach para cada una de las siete escalas del instrumento.

Con relación al tercer objetivo, se realizaron análisis de comparación de las puntuaciones de los participantes por género, edad y grado escolar. Para verificar el supuesto de normalidad se realizó la prueba de Kolmogorov-Smirnov, resultando que las siete subescalas del instrumento presentaron una diferencia significativa con respecto a la distribución normal, tal como se detallará en el apartado de resultados. Debido a ello, se decidió utilizar pruebas no paramétricas. Se empleó la prueba de Mann-Whitney para identificar posibles diferencias de género. Para los análisis por edad y por grado escolar, en cada caso, se utilizó la prueba de Kruskal-Wallis, y se complementó la misma con análisis post hoc de comparaciones múltiples.

Todos los cálculos se realizaron empleando los programas estadísticos IBM SPSS (versión 21) e IBM SPSS AMOS (versión 21). 


\section{Resultados}

Una vez realizado el análisis factorial confirmatorio se encontró que todas las cargas factoriales resultaron estadísticamente significativas y, como se puede observar en la figura 1, sus valores estandarizados (standardized regression weights) oscilaron entre .43 (valor más bajo) y .82 (valor más alto). Por su parte, a excepción del reactivo 6, todos los coeficientes de determinación estandarizados (squared multiple correlations, $r^{2}$ ) se ubicaron por encima de .20, siendo el mayor de .67 (reactivo 21). Los índices de bondad de ajuste obtenidos fueron los siguientes: $\mathrm{Ji}^{2} / \mathrm{gl}=3.602$, RMSEA $=.051$, $\mathrm{SRMR}=.0588, \mathrm{GFI}=.911, \mathrm{AGFI}=.893, \mathrm{PGFI}=.758, \mathrm{NFI}=.871, \mathrm{TLI}=.890$, y CFI $=.903$.

En la tabla 2 se muestran los índices de bondad de ajuste obtenidos para este modelo, así como sus valores de referencia. Como se puede apreciar, seis de los índices considerados se colocaron dentro del rango apropiado (Chi-cuadrado normado, RMSEA, SRMR, GFI, CFI y PGFI). Por su parte, los índices AGFI, NFI y TLI presentaron valores abajo del criterio estipulado, aunque cercanos a este, lo que significa una aceptabilidad marginal.

Pasando al análisis de la confiabilidad, los índices alfa de Cronbach para las escalas correspondientes a Estrategias agresivas, Denunciar, Ignorar y desviar la preocupación, Buscar apoyo, Reunir pruebas, Evitar al agresor y Desconexión fueron de .838, $.844, .724, .824, .765, .707$ y .701 . Para el instrumento total (31 reactivos) el alfa de Cronbach fue de .837 .

Las puntuaciones obtenidas por los participantes en cada una de las escalas (media y desviación estándar), así como los valores de asimetría y curtosis, se presentan en la tabla 3. Como se puede observar, la prueba de Kolmogorov-Smirnov resultó ser estadísticamente significativa en todos los casos, lo cual indica una distribución de los datos que se aleja de la distribución normal.

Por otra parte, los resultados del análisis de las diferencias de género se presentan en la tabla 4. Como puede observarse, las mujeres obtuvieron puntuaciones más altas que los hombres de manera estadísticamente significativa en las estrategias de Denunciar, Buscar apoyo, Reunir pruebas, Evitar al agresor y Desconexión; mientras que los varones obtuvieron puntuaciones más altas en Estrategias agresivas, de manera estadísticamente significativa.

En cuanto a las diferencias por grupos de edad, en la tabla 5 se presentan los resultados obtenidos en la prueba Kruskall-Wallis. Como se puede apreciar, solamente resultó estadísticamente significativa la relativa a las Estrategias agresivas, observándose una tendencia al aumento de dicha puntuación. En la prueba post hoc de comparaciones múltiples se ubicó la diferencia significativa entre el grupo de 12-15 años y los otros dos: con el de 16-17 ( $\not<.05)$ y el de 18 a 20 años $(\not<.05)$. 


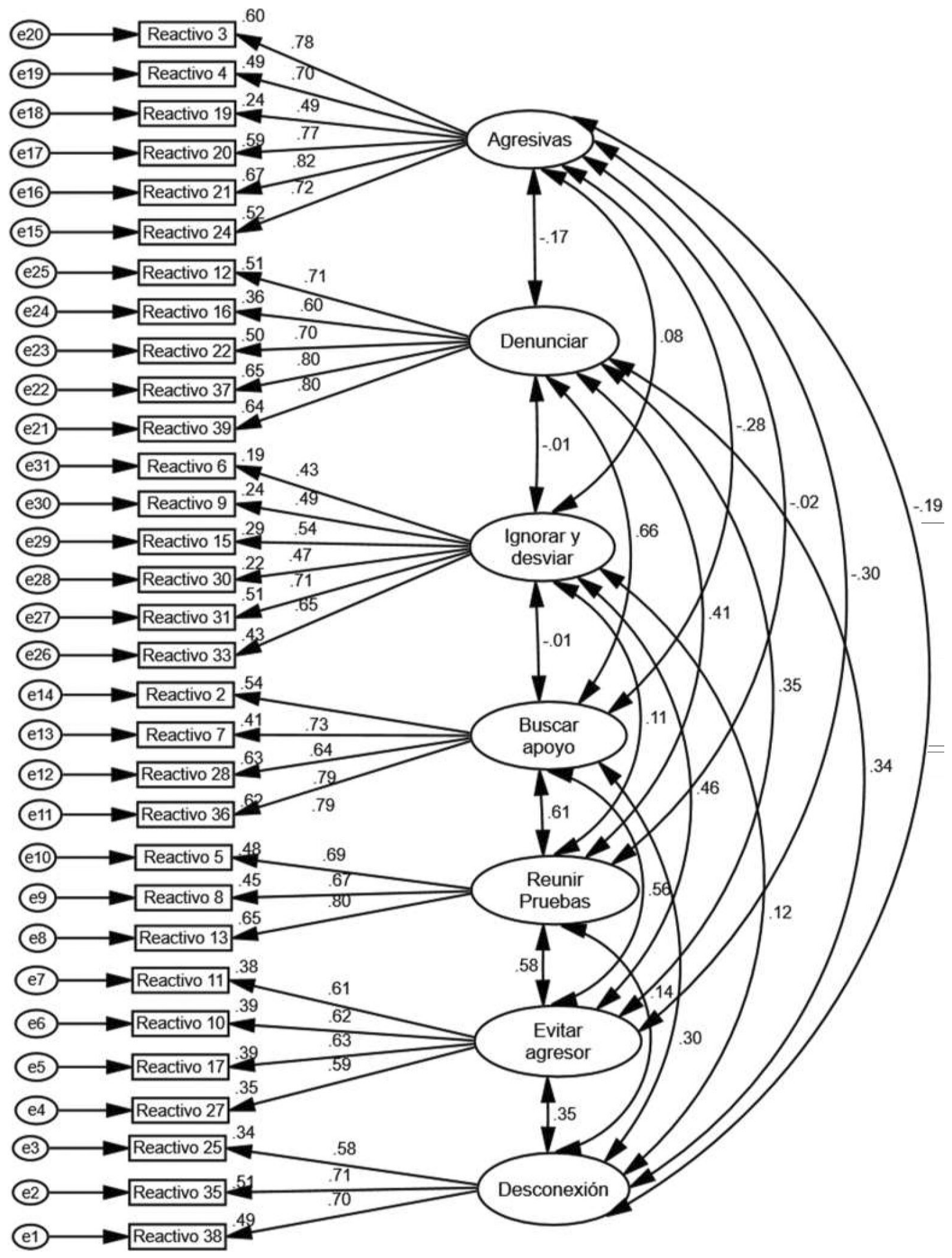

Figura 1. Resultados del análisis factorial confirmatorio del

Inventario de Estrategias de Afrontamiento del Ciberacoso desde la perspectiva de la Víctima.

$N=$ 992. Los valores sobre las flechas unidireccionales representan las cargas factoriales estandarizadas, los números sobre las puntas de flecha a la izquierda del gráfico representan los coeficientes de determinación estandarizados. Los valores sobre las flechas bidireccionales corresponden a los coeficientes de correlación entre los factores. 
Tabla 2. Índices de bondad de ajuste obtenidos y sus valores de referencia.

\begin{tabular}{lccc}
\multicolumn{1}{c}{ Índices } & Valores aceptables & Valores obtenidos & Aceptabilidad \\
\hline Indicadores del ajuste absoluto & & & \\
\hline Chi-cuadrado & No estadísticamente significativo & $1487.681(\mathrm{gl}=413), p<.001$ & No aceptable \\
\hline GFI & Mayor que $.90^{1,2}$ & .911 & Aceptable \\
\hline RMSEA & Entre .05 y $.08^{2}$ & .051 & Aceptable \\
\hline SRMR & Entre .05 y $.075^{2}$ & 0.588 & Aceptable \\
\hline Indices de ajuste incremental & & & Marginal \\
\hline NFI & Mayor que $.90^{1,2}$ & .871 & Aceptable \\
\hline CFI & Mayor que $.90^{1,2}$ & .903 & Marginal \\
\hline TLI & Mayor que $.90^{1,2}$ & .890 & Marginal \\
\hline AGFI & Mayor que $.90^{1,2}$ & .893 & Aceptable \\
\hline Ajuste de parsimonia & & & Aceptable \\
\hline PGFI & Mayor que $.50^{3}$ & .758 & 3.602 \\
\hline Chi-cuadrado normada & Entre 3 y $5^{1}$ & &
\end{tabular}

${ }^{1}$ Hair et al. (1999), ${ }^{2}$ Moral de la Rubia (2016), ${ }^{3}$ Byrne (2010).

Fuente: Construcción personal.

Tabla 3. Estadísticos descriptivos y análisis de la normalidad de las escalas del Inventario de Estrategias de Afrontamiento del Ciberacoso desde la perspectiva de la Víctima.

\begin{tabular}{lcccc} 
& $\mathrm{M}(\mathrm{DE})$ & Asimetría $(\mathrm{EE})$ & Curtosis (EE) & Z \\
\hline Estrategias agresivas & $1.73(0.73)$ & $1.08(0.78)$ & $0.56(0.16)$ & $5.12^{* *}$ \\
\hline Denunciar & $2.36(0.83)$ & $0.11(0.78)$ & $-0.91(0.16)$ & $2.72^{* *}$ \\
\hline Ignorar y desviar la preocupación & $2.12(0.63)$ & $0.31(0.78)$ & $-0.24(0.16)$ & $2.60^{* *}$ \\
\hline Buscar apoyo & $3.14(0.82)$ & $-0.85(0.78)$ & $-0.21(0.16)$ & $4.88^{* *}$ \\
\hline Reunir pruebas & $3.30(0.76)$ & $-1.06(0.78)$ & $0.53(0.16)$ & $6.24^{* *}$ \\
\hline Evitar al agresor & $2.93(0.76)$ & $-0.54(0.78)$ & $-0.41(0.16)$ & $4.03^{* *}$ \\
\hline Desconexión & $1.81(0.73)$ & $0.72(0.78)$ & $-0.93(0.16)$ & $4.45^{* *}$ \\
\hline
\end{tabular}

$N=992$.

$\mathrm{M}=$ Media aritmética, $\mathrm{DE}=$ Desviación estándar, $\mathrm{EE}=$ error estándar. $\mathrm{Z}=\mathrm{Z}$ de Kolmogorov-Smirnov. $* * * p<.001$.

Fuente: Construcción personal.

En cuanto a las diferencias por grado escolar, en la tabla 6 se presentan los resultados obtenidos en la prueba Kruskall-Wallis. Como se puede apreciar, se encontraron diferencias estadísticamente significativas en cinco de las siete estrategias (las excepciones fueron las estrategias de Denunciar y de Ignorar y desviar la preocupación). En las Estrategias agresivas, el análisis post hoc ubicó esas diferencias en el grupo de primer año de secundaria en relación con los tres de bachillerato: primero $(\phi<.05)$, segundo $(\phi<.05)$ y tercero $(\phi<.05)$ años. En la estrategia de Buscar 
Tabla 4. Diferencias de género en estrategias de afrontamiento del ciberacoso.

Rango promedio

\begin{tabular}{lccc} 
& Hombres $(n=429)$ & Mujeres $(n=563)$ & U de Mann-Whitney \\
\hline Estrategias agresivas & 605.54 & 413.41 & $73984.00^{* * *}$ \\
\hline Denunciar & 457.68 & 526.08 & $104108.00^{* * *}$ \\
\hline Ignorar y desviar la preocupación & 509.62 & 486.50 & 115134.50 \\
\hline Buscar apoyo & 419.84 & 554.92 & $87874.50^{* * *}$ \\
\hline Reunir pruebas & 447.98 & 533.48 & $99946.50^{* * *}$ \\
\hline Evitar al agresor & 430.74 & 546.61 & $92551.00^{* * *}$ \\
\hline Desconexión & 436.13 & 542.50 & $94863.00^{* * *}$ \\
\hline
\end{tabular}

$N=992 .{ }^{* * *} p<.001$.

Fuente: Construcción personal.

Tabla 5. Diferencias por grupos de edad en estrategias de afrontamiento del ciberacoso.

\begin{tabular}{|c|c|c|c|c|}
\hline & & ango promedi & & \\
\hline & $\begin{array}{c}18 \text { a } 20 \text { años } \\
(n=174)\end{array}$ & $\begin{array}{c}18 \text { a } 20 \text { años } \\
(n=174)\end{array}$ & $\begin{array}{c}18 \text { a } 20 \text { años } \\
(n=174)\end{array}$ & $\begin{array}{c}\text { Chi-cuadrado } \\
\quad(g l=2)\end{array}$ \\
\hline Estrategias agresivas & 526.77 & 526.77 & 526.77 & $8.91 *$ \\
\hline Denunciar & 470.16 & 470.16 & 470.16 & 2.46 \\
\hline Ignorar y desviar la preocupación & 496.60 & 496.60 & 496.60 & 2.93 \\
\hline Buscar apoyo & 483.92 & 483.92 & 483.92 & 3.22 \\
\hline Reunir pruebas & 517.80 & 517.80 & 517.80 & 1.28 \\
\hline Evitar al agresor & 510.95 & 510.95 & 510.95 & 2.05 \\
\hline Desconexión & 481.02 & 481.02 & 481.02 & 0.69 \\
\hline
\end{tabular}

$N=992 . * * * p<.05$.

Fuente: Construcción personal.

Tabla 6. Diferencias por grado escolar en estrategias de afrontamiento del ciberacoso.

\begin{tabular}{|c|c|c|c|c|c|c|c|}
\hline & \multicolumn{6}{|c|}{ Rango promedio } & \multirow[b]{3}{*}{$\begin{array}{c}\text { Chi-cuadrado } \\
(g l=5)\end{array}$} \\
\hline & \multicolumn{3}{|c|}{ Secundaria } & \multicolumn{3}{|c|}{$\overline{\text { Bachillerato }}$} & \\
\hline & $\begin{array}{l}\text { 1er. año } \\
(n=65)\end{array}$ & $\begin{array}{l}\text { 2do. año } \\
(n=67)\end{array}$ & $\begin{array}{l}\text { 3er. año } \\
(n=66)\end{array}$ & $\begin{array}{l}\text { 1er. año } \\
(n=286)\end{array}$ & $\begin{array}{l}2 \text { do. año } \\
(n=246)\end{array}$ & $\begin{array}{l}\text { 3er. año } \\
(n=262)\end{array}$ & \\
\hline Estrategias agresivas & 394.12 & 414.35 & 436.89 & 519.79 & 514.15 & 515.93 & $21.033^{* *}$ \\
\hline Denunciar & 536.71 & 550.85 & 516.46 & 487.44 & 512.78 & 462.20 & 8.89 \\
\hline Ignorar y desviar la preocupación & 452.25 & 470.91 & 439.98 & 498.43 & 518.26 & 505.72 & 6.40 \\
\hline Buscar apoyo & 525.20 & 601.57 & 557.20 & 463.07 & 507.22 & 473.65 & $18.92^{* *}$ \\
\hline Reunir pruebas & 421.26 & 552.19 & 548.45 & 465.11 & 504.05 & 515.02 & $14.80^{*}$ \\
\hline Evitar al agresor & 489.99 & 577.01 & 547.39 & 459.77 & 497.06 & 504.27 & $12.44^{*}$ \\
\hline Desconexión & 513.58 & 585.03 & 441.52 & 489.93 & 518.47 & 470.02 & $13.33^{*}$ \\
\hline
\end{tabular}

$N=992 .{ }^{* * *} p<.001$. 
apoyo, el análisis post hoc mostró diferencia entre el segundo año de secundaria y el primero de bachillerato $(p<01)$, así como entre segundo de secundaria y tercer año de bachillerato $(p<.05)$. En Reunir pruebas el análisis post hoc no mostró diferencias estadísticamente significativas entre ninguna de las parejas comparadas. En Evitar al agresor, la diferencia significativa se ubicó entre el segundo año de secundaria y el primer año de bachillerato $(\phi<.05)$. Finalmente, en Desconexión, el análisis post hoc, segundo y tercer año de secundaria $(p<.05)$, así como entre el segundo año de secundaria y el tercer año de bachillerato $(p<.05)$.

\section{Discusión}

Tal como se explicó, en el presente trabajo se planearon tres objetivos específicos: a) realizar un análisis factorial confirmatorio del IEAC-V, con el fin de evaluar su estructura factorial y su bondad de ajuste; b) estudiar la confiabilidad de cada una de las escalas del instrumento, y c) analizar posibles diferencias significativas por género y edad.

Con relación al primer objetivo, una vez realizado el análisis factorial confirmatorio, las cargas factoriales y coeficientes de determinación resultaron todos ser adecuados, lo cual es un dato a favor de la validez de la estructura factorial del modelo. Con respecto a la bondad de ajuste del modelo a los datos, cabe observar que se obtuvo un ajuste adecuado de acuerdo a seis de los índices considerados: Chi-cuadrado normado, RMSEA, SRMR, GFI, CFI y PGFI. Estos índices figuran actualmente entre los más importantes citados en la literatura. En cuanto a los índices AGFI, NFI y TLI, sus valores estuvieron cercanos a .90, lo que indica que, si bien no alcanzaron el nivel crítico, en conjunto con todos los otros resultados mencionados aportan, no obstante, información a favor de la bondad de ajuste del modelo. De acuerdo con diversos autores, al existir diversidad de índices, la valoración final debe ponderar varios de ellos en conjunto (Byrne, 2010; Ferrando y Anguiano-Carrasco, 2010; Hair et al., 1999).

Con respecto al hecho de que el estadístico de Chi-cuadrado resultó ser estadísticamente significativo, indicando con ello una diferencia entre los datos y el modelo propuesto, cabe reiterar lo ya señalado en el sentido de que se trata de un indicador muy sensible al tamaño de la muestra, que tiende a resultar significativo en muestras grandes especialmente si su tamaño es mayor de 200 participantes (Hair et al., 1999); por tal motivo, no constituye un dato en contra de la bondad de ajuste del modelo en el presente trabajo. De cualquier manera, se recomienda realizar más estudios que generen información que pudiera contribuir a robustecer los hallazgos obtenidos.

Pasando al análisis de la confiabilidad, el criterio clásico de Nunnally (1978, citado en Rodríguez-Rodríguez y Reguant-Álvarez, 2020, p. 10) establece como aceptables valores alfa de Cronbach superiores a .70, lo cual se cumplió para todas las escalas 
del instrumento en el presente estudio. Incluso se obtuvieron valores alfa superiores a .80 en tres escalas (Estrategias agresivas, Denunciar, Buscar apoyo) y en el instrumento total (31 reactivos), lo cual indica un nivel bueno de confiabilidad (George y Mallery, 2003).

Con relación al tercer objetivo, las mujeres obtuvieron puntuaciones más altas en las estrategias de Denunciar, Buscar apoyo, Reunir pruebas y Evitar al agresor; mientras que los hombres obtuvieron valores más elevados en Estrategias agresivas. Por un lado, este resultado coincide con otros estudios en los que se ha documentado de manera consistente una mayor inclinación de las mujeres hacia estrategias positivas o asertivas (De la Caba y López, 2013; Luna-Bernal et al., 2020); por otro lado, aunque actualmente no existen resultados concluyentes en lo que refiere a las diferencias de género en la literatura sobre ciberagresión escolar (Álvarez-García, Barreiro-Collazo y Núñez, 2017), sí existen estudios en que se ha mostrado una mayor orientación de los hombres hacia la conducta agresiva on line (Yudes, Rey y Extremera, 2019).

En cuanto a las diferencias de edad, se observó una tendencia al aumento de las Estrategias agresivas, especialmente al comparar el grupo de 12 a 15 años con los otros dos. Este resultado coincide con el trabajo de revisión de la literatura llevado a cabo por Pérez Estrada (2019), en el que se encontró que "los estudiantes mayores son más propensos a utilizar estrategias de externalización, como maldecir en voz alta o defenderse sin recurrir a la agresión física (gritar, por ejemplo, o meterse en una discusión) o acosar a otra persona” (p. 26). Este resultado se ve robustecido en el presente estudio con el análisis por grado escolar, en el que se encontró que el grupo de primer año de secundaria presentó puntajes más bajos en relación con los tres de bachillerato en las estrategias agresivas. No obstante lo anterior, debe considerarse que en la literatura subsisten resultados divergentes con relación al papel de la variable edad en el afrontamiento durante la adolescencia (Coppari, Barcelata, Bagnoli, Codas, López y Martínez, 2019), por lo cual se aconseja en futuros estudios analizar de manera sistemática esa relación.

Finalmente, con respecto al análisis por grado escolar, además de lo señalado en el párrafo anterior, llama la atención que el grupo de segundo año de secundaria presentó una tendencia a puntuaciones más altas que los grados de bachillerato en las estrategias de Buscar apoyo, Evitar al agresor y Desconexión. Al respecto cabe mencionar que en la actualidad existe una gran escasez de estudios que comparen estrategias de afrontamiento del ciberacoso en secundaria y bachillerato.

Por un lado, este resultado coincide el trabajo de Vega, González-Pérez y Quintero-Vega (2013) realizado con estudiantes de secundaria de Tlaquepaque, Jalisco, en el que se encontró que "la principal elección para dar a conocer -siempre o con frecuencia- su condición de víctima fue la familia, principalmente los padres $(48,2 \%)$, seguido por los hermanos (40,8\%)" (p. 16), así como de los amigos, otros coetáneos 
y compañeros de escuela; ello debido a que la escala de Buscar apoyo en el presente estudio abarca reactivos que hacen alusión a buscar ayuda y soporte en un familiar o una persona de confianza. Para contextualizar adecuadamente el uso de este tipo de estrategia es importante considerar la situación de indefensión en que, por definición, se encuentra la víctima de ciberacoso. De acuerdo con Nacimiento y Mora-Merchán (2014), las víctimas "se caracterizan por intentar de manera repetida y formas diversas salir de la situación de acoso en la que se ven envueltas, sin desarrollar realmente recursos que les permitan salir de la dinámica en la que se encuentran” (p. 126). Ello vuelve altamente relevante la búsqueda de ayuda como forma adecuada de manejo del problema. A su vez, Evitar al agresor (orientada a utilizar medios tecnológicos y no tecnológicos para lograr no tener contacto con los agresores, sin necesidad de dejar de usar internet ni los sitios web de preferencia) se relaciona con esto último ya que, de acuerdo con Machmutow et al. (2012), confrontar al acosador, aunque sea de manera pacífica, puede constituir una experiencia que afecta negativamente al bienestar, sobre todo si se considera que por definición la situación se caracteriza por un desequilibrio de poder. Por tanto, los resultados del presente estudio podrían sugerir una tendencia a un mayor empleo de estas estrategias de tipo asertivo u orientadas al problema en los estudiantes de segundo grado de secundaria con respecto a los de bachillerato.

Por otro lado, sin embargo, este hallazgo contrasta con el hecho de que el grupo de segundo de secundaria presentó también una cierta tendencia hacia la estrategia de Desconexión mayor que los grados de tercero de secundaria y tercero de bachillerato. Como se mencionó, esta estrategia consiste en evadir la situación evitando completamente el uso de internet o de las páginas o sitios web relacionados con el problema. Tomando en cuenta la importancia que actualmente tienen las redes sociales y el Internet en la vida cotidiana de los adolescentes, dicha desconexión puede entenderse como una estrategia evasiva. Como se explicó, diversos estudios han mostrado que los individuos que utilizan el afrontamiento orientado al problema en situaciones de acoso cibernético tienden a tener resultados más positivos y adaptativos con respecto a quienes usan estrategias situadas en la emoción o de evitación (Alipan, Skues y Theiler, 2018; Ronis y Slaunwhite, 2019); por lo cual el uso de esta estrategia podría tener consecuencias negativas para el bienestar de los adolescentes.

Estos hallazgos contrastantes invitan a profundizar en estudios posteriores acerca de la cuestión de cómo se interrelacionan las diversas estrategias de afrontamiento de ciberacoso, y las posibles diferencias entre los adolescentes de secundaria y bachillerato, considerando sobre todo que en la actualidad hay una gran escasez de estudios orientados específicamente a comparar ambos niveles educativos. Un estudio reciente de Sánchez-Domínguez, Magaña y Pozo (2020), por ejemplo, mostró una mayor prevalencia de ciberacoso en bachillerato, pero una mayor presencia de casos 
con duración mayor a seis meses en secundaria. Por tanto, el desarrollo de estudios orientados sistemáticamente a evaluar las diferencias por grado escolar podría ser una vía de estudio importante para un mayor entendimiento de estos fenómenos.

En conclusión, el presente estudio proporciona información relevante a favor de la validez y confiabilidad del instrumento IEAC-V, desde una perspectiva confirmatoria. En particular, muestra como plausible el modelo de siete factores relacionados como una configuración subyacente a los 31 reactivos que componen dicho inventario. Estos siete factores, a su vez, representan siete diferentes tipos de estrategias utilizadas por los adolescentes para hacer frente a las situaciones de ciberacoso, por lo cual puede considerarse que el presente trabajo no solo tiene un valor instrumental sino que sus resultados también contribuyen a un mejor entendimiento de las maneras en que los adolescentes hacen frente a este tipo de situaciones.

De acuerdo con los hallazgos obtenidos, puede considerarse al IEAC-V como un instrumento válido y confiable para medir estrategias de afrontamiento del ciberacoso en población adolescente mexicana escolarizada de nivel secundaria (educación media básica) y bachillerato (educación media superior), ya que tales fueron las delimitaciones del presente trabajo. Por ello se recomienda para estudios posteriores generar información acerca de si el instrumento conserva sus propiedades en otro tipo de muestras, como pudieran ser los jóvenes de nivel universitario (sujetos a otro tipo de dinámicas de interacción escolar) o los adolescentes de otros contextos culturales (p. e., de otro país con diferencias culturales observables). También se recomienda para futuros estudios generar información acerca de la validez convergente y divergente del instrumento, examinando sus relaciones con otras variables psicológicas y psicosociales de relevancia.

\section{ReferenCias}

Alipan, A., Skues, J. L., y Theiler, S. (2018). “They will find another way to hurt you": Emerging adults' perceptions of coping with cyberbullying. Emerging Adulthood, 1-13. https://doi.org/10.1177/2167696818816896.

Álvarez-García, D., Barreiro-Collazo, A., y Núñez, J.-C. (2017). Ciberagresión entre adolescentes: prevalencia y diferencias de género. Comunicar, 25(50), 89-97. https://doi.org/10.3916/C50-2017-08.

Baldry, A. C., Farrington, D., y Sorrentino, A. (2015). Am I at risk of cyberbullying? A narrative review and conceptual framework for research on risk of cyberbullying and cybervictimization: The risk and needs assessment approach. Aggression and Violent Behavior, (23), 36-51. https://doi.org/10.1016/j. avb.2015.05.014.
Batista-Foguet, J. M., Coenders, G., y Alonso, J. (2004). Análisis factorial confirmatorio. Su utilidad en la validación de cuestionarios relacionados con la salud. Medicina Clínica, 122(supl. 1), 21-27.

Beltrán-Catalán, M., Zych, I., Ortega-Ruiz, R., y Llorent, V. (2018). Victimisation through bullying and cyberbullying: Emotional intelligence, severity of victimization and technology use in different types of victims. Psicothema, 30(2), 183-188. https://doi. org/10.7334/psicothema2017.313.

Bezerra Souza, S., Veiga Simao, A. M., y Caetano, A. P. (2014). Cyberbullying: Percepcoes acerca do fenomeno e das estratégias de enfrentamento. Psicología: Reflexao e Crítica, 27(3), 582-590. http://dx.doi. org/10.1590/1678-7153.201427320. 
Byrne, B. M. (2010). Structural equation modeling with AMOS. Basic concepts, applications, and programming (2a. ed.). Taylor and Francis.

Coppari, N., Barcelata, B., Bagnoli, L., Codas, G., López Humada, H., y Martínez Cañete, U. (2019). Influencia del sexo, edad y cultura en las estrategias de afrontamiento de adolescentes paraguayos y mexicanos. Universitas Psychologica, 18(1), 1-13. https://doi.org/10.11144/ Javeriana.upsy18-1.isec.

De Gante-Casas, A., Luna-Bernal, A. C. A., López-Aguilar, R. M., y Nava-Preciado, J. M. (2020). Diseño y estudio psicométrico de un inventario para evaluar estrategias de afrontamiento del ciberbullying desde la perspectiva del observador. Revista Educación y Desarrollo, (52), 37-48.

De la Caba Collado, M. A., y López Atxurra, R. (2013). La agresión entre iguales en la era digital: estrategias de afrontamiento de los estudiantes del último ciclo de primaria y del primero de secundaria. Revista de Educación, (362), 247-272. http://dx.doi.org/10.4438/1988-592XRE-2011-362-160.

Del Rey, R., Estévez, M., y Ojeda, M. (2018). El ciberacoso y su respuesta educativa. En E. Jiménez, M. Garmendia y M. A. Casado (coords.), Entre selfies y whatsapps. Oportunidades y riesgos para la infancia y la adolescencia conectada (pp. 125-139). Gedisa.

Elizalde Castillo, A. (2010). Estudio descriptivo de las estrategias de afrontamiento del bullying en profesorado mexicano. Electronic Journal of Research in Educational Psychology, 8(1), 353-372.

Escobedo-Portillo, M. T., Hernández-Gómez, J. A., Estebané-Ortega, V., y Martínez-Moreno, G. (2016). Modelos de ecuaciones estructurales: características, fases, construcción, aplicación y resultados. Ciencia y Trabajo, 18(55), 16-22. http://dx.doi.org/10.4067/ S0718-24492016000100004.

Ferrara, P., Ianniello, F., Villani, A., y Corsello, G. (2018). Cyberbullying a modern form of bullying: Let's talk about this health and social problem. Italian Journal of Pediatrics, 44(1), 14. https://doi.org/10.1186/s13052018-0446-4.

Ferrando, P. J., y Anguiano-Carrasco, C. (2010). El análisis factorial como técnica de investigación en psicología. Papeles del Psicólogo, 31(1), 18-33.

Garaigordobil Landazábal, M. (2011). Prevalencia y consecuencias del ciberbullying: una revisión internacional.
Journal of Psychology \& Psychological Therapy, 11(2), 233-254.

Garaigordobil Landazabal, M. (2013). Screening de acoso entre iguales. TEA Ediciones.

George, D., y Mallery, P. (2003). SPSS for Windows step by step: $A$ simple guide and reference. 11.0 update (4a. ed.). Allyn \& Bacon.

Giménez Gualdo, A. M. (2015). Estrategias de afrontamiento ante el cyberbullying. Una mirada cualitativa desde la perspectiva de los escolares. Campo Abierto. Revista de Educación, 1(1), 49-65.

Gradinger, P., Yanagida, T., Strohmeier, D., y Spiel, C. (2015). Prevention of cyberbullying and cybervictimization: Evaluation of the ViSC social competence program. Journal of School Violence, 14(1), 87-110. https://doi.org/10.1080/15388220.2014.963231.

Hair, J., Anderson, R., Tatham, R., y Black, W. (1999). Análisis multivariante (trad. E. Prentice y D. Cano). Prentice Hall Iberia.

Herrera-López, M., Romera, E., y Ortega-Ruiz, R. (2017). Bullying y cyberbullying en Colombia; coocurrencia en adolescentes escolarizados. Revista Latinoamericana de Psicologia, 49(3), 163-172. https:// doi.org/10.1016/j.rlp.2016.08.001.

Herrera-López, M., Romera, E. M., y Ortega-Ruiz, R. (2018). Bullying y cyberbullying en Latinoamérica: un estudio bibliométrico. Revista Mexicana de Investigación Educativa, 23(76), 125-155.

Jacobs, N. C. L., Vollink, T., Dehue, F., y Lechner, L. (2015). The development of a self-report questionnaire on coping with cyberbullying: The Cyberbullying Coping Questionnaire. European Societies, 5(2), 460-491. https://doi.org/10.3390/soc5020460.

Lazarus, R. S., y Folkman, S. (1984). Stress, appraisal, and coping. Springer Publishing Company.

Lee, C., y Shin, N. (2017). Prevalence of cyberbullying and predictors of cyberbullying perpetration among Korean adolescents. Computers in Human Behavior, (68), 352-358. https://doi.org/10.1016/j. chb.2016.11.047.

León, B., Felipe, E., Fajardo, F., y Gómez-Carroza, T. (2012). Cyberbullying en una muestra de estudiantes de educación secundaria: variables moduladoras y redes sociales. Electronic Journal of Research in Educational Psychology, 10(27), 771-788. 
Li, Q. (2008). A cross-cultural comparison of adolescents' experience related to cyberbullying. Educational Research, 50(3), 223-234. https://doi. org/10.1080/00131880802309333.

Lucio López, L. (2014). Cyberbullying en alumnos del nivel medio superior [Tesis de Licenciatura]. Universidad Autónoma del Estado de México. Repositorio Institucional UAEM. Recuperado de: http://hdl.handle. net/20.500.11799/49383.

Lucio López, L. A., y Gómez Triana, F. J. (2018). Las redes sociales como campo de batalla, el cyberbullying en estudiantes del nivel medio superior. En A. SánchezCastañeda (coord.), Acoso escolar y cyberbullying. Retos, prevención y sensibilización (pp. 105-115). Universidad Nacional Autónoma de México, Defensoría de los Derechos Universitarios.

Luna-Bernal, A. C. A., Gómez-Pérez, M. A., SandovalMartínez, J., y Valencia-Aguirre, A. C. (2020). Estructura factorial y confiabilidad de un inventario para evaluar estrategias de afrontamiento del cyberbullying desde la perspectiva de la víctima. Revista Educación y Desarrollo, (53), 71-81.

Machackova, H., Cerna, A., Sevcikova, A., Dedkova, L., y Daneback, K. (2013). Effectiveness of coping strategies for victims of cyberbullying. Cyberpsychology: Journal of Psychosocial Research on Cyberspace, 7(3). http:// dx.doi.org/10.5817/CP2013-3-5.

Machmutow, K., Perren, S., Sticca, F., y Alsaker, F. D. (2012). Peer victimisation and depressive symptoms: Can specific coping strategies buffer the negative impact of cybervictimisation? Emotional and Behavioural Difficulties, 17(3-4), 403-420. https://doi.org/10.108 0/13632752.2012.704310.

Madrid López, E. J., Valdéz Cuervo, A. A., y Vera Noriega, J. A. (2015, nov. 16). Diferencias en los estilos de afrontamiento de estudiantes victimas y no involucrados en el bullying. Ponencia presentada en el XIII Congreso Nacional de Investigación Educativa, Chihuahua, México. Recuperado de: http://www.comie.org.mx/ congreso/memoriaelectronica/v13/doc/0133.pdf.

Mallmann, C. L., Macedo Lisboa, C. S., y Zanatta Calza, T. (2018). Cyberbullying and coping strategies in adolescents from Southern Brazil. Acta Colombiana de Psicología, 21(1), 13-43. http://dx.doi.org/10.14718/ acp.2018.21.1.2.
Martínez-Vilchis, R., Pozas, J., Jiménez, K., Morales, M., Miranda, D., Delgado, M., y Cuenca, V. (2015). Prevención de la violencia cara a cara y virtual en el bachillerato. Psychology, Society \& Education, 7(2), 201-212.

Matos, A., Vieira, C., Amado, J., Pessoa, T., y Martins, M. J. (2018). Cyberbullying in Portuguese schools: Prevalence and characteristics. Journal of School Violence, 17(1), 123-137. https://doi.org/10.1080/15388220. 2016.1263796.

Medrano, L. A., y Muñoz-Navarro, R. (2017). Aproximación conceptual y práctica a los modelos de ecuaciones estructurales. Revista Digital de Investigación en Docencia Universitaria, 11(1), 219-239. https://doi. org/10.19083/ridu.11.486.

Moral de la Rubia, J. (2016). Análisis factorial y su aplicación al desarrollo de escalas. En R. Landero Hernández y M. T. González Ramírez (eds.), Estadística con SPSS y metodología de la investigación (pp. 387-443). Trillas.

Nacimiento Rodríguez, L., y Mora-Merchán, J. A. (2014). El uso de estrategias de afrontamiento y habilidades metacognitivas ante situaciones de bullying y cyberbullying. European Journal of Education and Psychology, 7(2), 121-129. https:// doi.org/10.30552/ejep.v7i2.104.

Ortega-Barón, J. y Carrascosa, L. (2018). Malestar psicológico y apoyo psicosocial en víctimas de ciberbullying. INFAD Revista de Psicología, 2(1), 357-366. http://doi.org/10.17060/ijodaep.2018.n1.v2.1241.

Ortega Ruiz, R., Del Rey, R., y Casas, J. (2016). Evaluar el bullying y el cyberbullying validación española del EBIP-Q. Psicología Educativa, 22(1), 71-79. https://doi. org/10.1016/j.pse.2016.01.004.

Palladino, B. E., Nocentini, A., y Menesini, E. (2012). Online and offline peer led models against bullying and cyberbullying. Psicothema, 24(4), 634-639.

Parris, L., Varjas, K., Meyers, J., y Cutts, H. (2012). High school students' perceptions of coping with cyberbullying. Youth \& Society, 44(2), 284-306. https://doi. org/10.1177/0044118X11398881.

Pérez Estrada, M. (2019). Revisión sistemática de las estrategias de afrontamiento de los estudiantes víctimas del acoso escolar [Trabajo de Maestría]. Universidad de Sevilla. Depósito de Investigación US. Recuperado de: https://hdl. handle.net/11441/93917.

Raskauskas, J., y Huynh, A. (2015). The process of coping with cyberbullying: A systematic review. $A g$ - 
gression and Violent Behavior, (23), 118-125. https:/ / doi. org/10.1016/j.avb.2015.05.019.

Redondo, J., Luzardo-Briceño, M., García-Lizarazo, K. L., e Inglés, C. J. (2017). Impacto psicológico del ciberbullying en estudiantes universitarios: un estudio exploratorio. Revista Colombiana de Ciencias Sociales, 8(2), 458478. http://dx.doi.org/10.21501/22161201.2061.

Rodríguez-Rodríguez, J., y Reguant-Álvarez, M. (2020). Calcular la fiabilidad de un cuestionario o escala mediante el SPSS: el coeficiente alfa de Cronbach. REIRE Revista d'Innovació $i$ Recerca en Educació, 13(2), 1-13. Recuperado de: http://revistes.ub.edu/index. $\mathrm{php} / \mathrm{REIRE} / \mathrm{article} / \mathrm{view} / 30048$.

Ronis, S., y Slaunwhite, A. (2019). Gender and geographic predictors of cyberbullying victimization, perpetration, and coping modalities among youth. Canadian Journal of School Psychology, 34(1), 3-21. https://doi. org/10.1177/0829573517734029.

Sánchez-Domínguez, J. P., Magaña Raymundo, L., y Pozo Osorio, M. C. (2020). Estudio comparativo del ciberacoso en escolares de secundaria y media superior. Revista Electrónica sobre Tecnología, Educación y Sociedad, 7(14), 39-52. https://www.ctes.org.mx/index.php/ ctes/article/view/715.

Schneider, S. K., O’Donnell, L., Stueve, A., y Coulter, R. W. (2012). Cyberbullying, school bullying, and psychological distress: a regional census of high school students. American Journal of Public Health, 102(1), 171177. https://doi.org/10.2105/AJPH.2011.300308.

Sléglová, V., y Cerna, A. (2011). Cyberbullying in adolescent victims: Perception and coping. Cyberpsychology. Journal of Psychosocial Research on Cyberspace, 5(2).

Smith, P. K., Mahdavi, J., Carvalho, M., Fisher, S., Russell, S., y Tippett, N. (2008). Cyberbullying: Its nature and impact in secondary school pupils. Journal of Child Psychology and Psychiatry, and Allied Disciplines, 49(4), 376-385. https://doi.org/10.1111/j.14697610.2007.01846.x.
Sticca, F., Machmutow, K., Stauber, A., Perren, S., Palladino, B. E., Nocentini, A., Menesini, E., Corcoran, L., y McGuckin, C. (2015). The Coping with Cyberbullying Questionnaire: Development of a new measure. Societies, (5), 515-536. https://doi.org/10.3390/ soc5020515.

Torres-Acuña, G. M., Rivera Hernández, C., y Navarro Rangel, Y. (2019). Validación de una escala para medir afrontamiento ante ciberagresiones entre universitarios. Revista Electrónica de Investigación Educativa, 21 (e09), 1-11. https://doi.org/10.24320/redie.2019.21. e09.1907.

Valdés Cuervo, A. A., Carlos Martínez, E. A., Tánori Quintana, J., y Wendlandt Amezaga, T. R. (2014). Differences in types and technological means by which Mexican high schools students perform cyberbullying: Its relationship with traditional bullying. Journal of Educational and Developmental Psychology, 4(1), 105-113. http://dx.doi.org/10.5539/jedp.v4n1p105.

Vega-Cauich, J. I. (2019). Prevalencia del bullying en México: un meta-análisis del bullying tradicional y cyberbullying. Diversitas, 15(1), 111-127. https://doi. org/10.15332/22563067.4020.

Vega López, M. G., González-Pérez, G. J., y QuinteroVega, P. P. (2013). Ciberacoso: victimización de alumnos en escuelas secundarias públicas de Tlaquepaque, Jalisco, México. Educación y Desarrollo, (25), 13-20.

Waasdorp, T., y Bradshaw, C. (2015). The overlap between cyberbullying and traditional bullying. Journal of Adolescent Health, 56(5), 483-488. https://doi. org/10.1016/j.jadohealth.2014.12.002.

Weber, N. L., y Pelfrey Jr., W. V. (2014). Cyberbullying: Causes, consequences, and coping strategies. LFB Scholarly Publishing LLC.

Yudes, C., Rey Peña, L., y Extremera Pacheco, N. (2019). Ciberagresión, adicción a internet e inteligencia emocional en adolescentes: un análisis de diferencias de género. Voces de la Educación, (esp. 2), 26-44.

Cómo citar este artículo:

Sandoval Martínez, J., Luna Bernal, A. C. A., y De Gante Casas, A. (2021). Estrategias de afrontamiento del ciberacoso desde la perspectiva de la víctima en adolescentes mexicanos de secundaria y bachillerato. IE Revista de Investigación Educativa de la REDIECH, 12, e1104. doi: 10.33010/ie_rie_rediech.v12i0.1104. 